\title{
Nilpotent semigroups for the characterization of flat outputs of discrete-time switched linear and LPV systems
}

\author{
Jérémy Parriaux ${ }^{1,2}$ and Gilles Millérioux ${ }^{1,2}$
}

\begin{abstract}
This paper addresses the problem of flat output characterization for switched linear systems along with an extension to LPV systems. A condition which has a polynomial complexity with respect to the dimension of the system and a practical approach for checking this condition are provided.
\end{abstract}

\section{INTRODUCTION}

Flatness is an important control-theoretical concept introduced in [1]. For a flat discrete-time system (linear or nonlinear), the state variable as well as the input to the system can be written as some function of the output (including forward and backward shifts in the output). Such a property is especially interesting both for state reconstruction and for control perspectives. As for the state reconstruction context, it is clear from the definition that flatness provides a generic way of reconstructing the state vector despite possibly unknown inputs. Even more is true, flatness is a structural property of a dynamical system and so provides conditions for the existence of an unknown input observer without any a priori structure of the observer. For control purposes, flatness is also relevant insofar as, from the definition again, it provides a constructive way of designing a feedforward control to track a prescribed trajectory of the plant output. An overview along with applications can be found in the book [2]. This being the case, an important issue related to flatness is the problem of checking whether a given output of a dynamical system is flat or not. An interesting approach has been proposed in [3] for continuous linear systems. Flat output characterization has been addressed for the first time for discrete-time switched linear systems in [4]. However, the condition was not, in general, tractable. The purpose of this paper is to give a new criterion for discrete-time switched linear systems with better tractability. It is based on the notion of nilpotent semigroups. The corresponding algorithm for checking the conditions is given. Furthermore, an extension to LPV systems is proposed. The layout is the following. In Section II, we recall some basics on flatness for switched linear systems. Section III is devoted to flat output characterization as well as the corresponding algorithm. An extension to LPV systems is proposed in Section IV. Finally, Section $\mathrm{V}$ is devoted to illustrative examples.

This work was partially supported by the CPER Région Lorraine (AOC Project), Fédération Charles Hermite, and INRIA

1 Université de Lorraine, CRAN, UMR 7039, ESSTIN, 2 rue Jean Lamour, Vandœuvre-lès-Nancy Cedex, 54506, France

2 CNRS, CRAN, UMR 7039, France

\section{PRELiminaries AND DEFinitions}

We shall examine throughout this paper switched linear systems in the form

$$
\left\{\begin{array}{l}
x_{k+1}=A_{\sigma(k)} x_{k}+B_{\sigma(k)} u_{k} \\
y_{k}=C_{\sigma(k)} x_{k}+D_{\sigma(k)} u_{k}
\end{array}\right.
$$

The state vector is $x_{k} \in \mathbb{R}^{n}$, the input is $u_{k} \in \mathbb{R}^{m}$ and the output is $y_{k} \in \mathbb{R}^{p}$. All the matrices, namely $A_{\sigma(k)} \in \mathbb{R}^{n \times n}$, $B_{\sigma(k)} \in \mathbb{R}^{n \times m}, C_{\sigma(k)} \in \mathbb{R}^{p \times n}$ and $D_{\sigma(k)} \in \mathbb{R}^{p \times m}$ belong to the respective finite sets of cardinality $J: \mathcal{A}=$ $\left\{A_{1}, \ldots, A_{J}\right\}, \mathcal{B}=\left\{B_{1}, \ldots, B_{J}\right\}, \mathcal{C}=\left\{C_{1}, \ldots, C_{J}\right\}$ and $\mathcal{D}=\left\{D_{1}, \ldots, D_{J}\right\}$. At a given time $k$, the mode is delivered by a switching function $\sigma: k \in \mathbb{N} \longmapsto \sigma(k) \in$ $\{1, \ldots, J\}=\mathcal{J}$. A sequence of modes (also called path) over an interval of time $\left[k_{1}, k_{2}\right]$, that is $\left\{\sigma\left(k_{1}\right), \ldots, \sigma\left(k_{2}\right)\right\}$, is denoted by $\{\sigma\}_{k_{1}: k_{2}}$. For a given switching rule $\sigma$, the set of corresponding mode sequences over an interval of time of length $T+1$ belongs to $\mathcal{J}^{T+1}$. This set has cardinality $J^{T+1}$ if $\sigma$ is arbitrary since, in this case, it is composed of all the possible mode sequences. Otherwise, the cardinality is lower because there may exist some forbidden patterns. Let $\mathcal{U}$ be the space of input sequences over the time interval $[0, \infty)$ and $\mathcal{Y}$ the corresponding output space. At time $k$, for each initial state $x_{k} \in \mathbb{R}^{n}$, when the system (1) is driven by the input sequence $\{u\}_{k: k+T}=\left\{u_{k}, \ldots, u_{k+T}\right\} \in \mathcal{U}$, for a mode sequence $\{\sigma\}_{k: k+T},\left\{x\left(x_{k}, \sigma, u\right)\right\}_{k: k+T}$ refers to the solution of (1) in the interval of time $[k, k+T]$ starting from $x_{k}$ and $\left\{y\left(x_{k}, \sigma, u\right)\right\}_{k: k+T} \in \mathcal{Y}$ refers to the corresponding output sequence in the same interval of time $[k, k+T]$.

We introduce the subsequent vectors and matrices. For any integer $n, \mathbf{1}_{n}$ refers to the $n$-dimensional identity matrix and $\mathbf{0}_{n \times m}$ stands for the $n \times m$ zero matrix. If irrelevant, the dimension of the zero matrix will be omitted and we shall merely write $\mathbf{0}$.

$$
\begin{array}{r}
u_{k: k+i}=\left(\begin{array}{c}
u_{k} \\
u_{k+1} \\
\vdots \\
u_{k+i}
\end{array}\right), y_{k: k+i}=\left(\begin{array}{c}
y_{k} \\
y_{k+1} \\
\vdots \\
y_{k+i}
\end{array}\right) \\
I_{m \times r}=\left(\begin{array}{ll}
\mathbf{1}_{m} & \mathbf{0}_{m \times(m \cdot r)}
\end{array}\right) \\
\mathcal{O}_{\sigma(k: k+i)}=\left(\begin{array}{c}
C_{\sigma(k)} \\
C_{\sigma(k+1)} A_{\sigma(k)} \\
\vdots \\
C_{\sigma(k+i)} A_{\sigma(k)}^{\sigma(k+i-1)}
\end{array}\right)
\end{array}
$$


The matrix $\mathcal{O}_{\sigma(k: k+i)}$ involves the transition matrix

$$
\begin{aligned}
A_{\sigma\left(k_{0}\right)}^{\sigma\left(k_{1}\right)} & =A_{\sigma\left(k_{1}\right)} A_{\sigma\left(k_{1}-1\right)} \cdots A_{\sigma\left(k_{0}\right)} \text { if } k_{1} \geq k_{0} \\
& =\mathbf{1}_{n} \text { if } k_{1}<k_{0}
\end{aligned}
$$

Finally, we recursively define the matrix

$$
M_{\sigma(k: k+i)}=\left(\begin{array}{cc}
D_{\sigma(k)} & \mathbf{0} \\
\mathcal{O}_{\sigma(k: k+i)} B_{\sigma(k)} & M_{\sigma(k+1: k+i)}
\end{array}\right)
$$

with

$$
M_{\sigma(k: k)}=D_{\sigma(k)}
$$

Let us notice that the notation $\sigma(k: k+i)$, which points out that the related matrix depends on the sequence $\{\sigma(k), \ldots, \sigma(k+i)\}$ is somehow abusive since $\sigma$ is defined over $\mathbb{N}$ and not over $\mathbb{N}^{i+1}$. However, since it does not induce confusion, such a notation will be used accordingly.

Flatness is closely related to the notion of left invertibility. Left invertibility for switched linear systems has been addressed in [5] for continuous-time systems and in [6], [4] for discrete-time systems.

Definition 1 ([6], [4]): A system is a left $r$-delay inverse for (1) if, under identical initial conditions $x_{0}$ and identical sequences $\{\sigma\}_{0: \infty}$, there exists a non negative integer $r$ such that, when driven by $y_{k: k+r}$, the equalities $\widehat{x}_{k+r}=x_{k}$ and $\widehat{u}_{k+r}=u_{k}$ hold for all $k \geq 0, \widehat{u}_{k}$ being its output at time $k$. The non negative integer $r$ is called the inherent delay.

Theorem 1 ([6], [4]): The system

$$
\left\{\begin{aligned}
\widehat{x}_{k+r+1}= & P_{\sigma(k: k+r)} \widehat{x}_{k+r} \\
& +B_{\sigma(k)} I_{m \times r}\left(M_{\sigma(k: k+r)}\right)^{\dagger} y_{k: k+r} \\
\widehat{u}_{k+r}= & -I_{m \times r}\left(M_{\sigma(k: k+r)}\right)^{\dagger} \mathcal{O}_{\sigma(k: k+r)} \widehat{x}_{k+r} \\
& +I_{m \times r}\left(M_{\sigma(k: k+r)}\right)^{\dagger} y_{k: k+r}
\end{aligned}\right.
$$

with

$$
P_{\sigma(k: k+r)}=A_{\sigma(k)}-B_{\sigma(k)} I_{m \times r}\left(M_{\sigma(k: k+r)}\right)^{\dagger} \mathcal{O}_{\sigma(k: k+r)}
$$

is a left $r$-delay inverse system for (1).

The matrix $\left(M_{\sigma(k: k+r)}\right)^{\dagger}$ is the classical Moore-Penrose generalized inverse of $M_{\sigma(k: k+r)}$. The matrices $P_{\sigma(k: k+r)}$ are called the left-inverse dynamical matrices.

Remark 1: In Definition 1, the initial condition is considered at the particular discrete time $k=0$ but can be replaced by any other initial condition $x_{k}$ considered at time $k$.

\section{A. Flatness}

Definition 2 ([4]): A square $(p=m)$ dynamical system is said to be flat if there exists a set of independent variables $y_{k}$, referred to as flat outputs, such that all system variables can be expressed as a function of the flat output and a finite number of its backward and/or forward shifts. In particular, there exist two functions $\mathcal{F}$ and $\mathcal{G}$ which obey

$$
\left\{\begin{array}{l}
x_{k}=\mathcal{F}\left(y_{k+k_{\mathcal{F}}}, \ldots, y_{k+k_{\mathcal{F}}^{\prime}}\right) \\
u_{k}=\mathcal{G}\left(y_{k+k_{\mathcal{G}}}, \ldots, y_{k+k_{\mathcal{G}}^{\prime}}\right)
\end{array}\right.
$$

where $k_{\mathcal{F}}, k_{\mathcal{F}}^{\prime}, k_{\mathcal{G}}$ and $k_{\mathcal{G}}^{\prime}$ are $\mathbb{Z}$-valued integers. Considering the expression of $u_{k}$ in (7), it is clear that left invertibility is a necessary condition for flatness.
The issue of flat output characterization consists in checking whether a given output of a dynamical system is flat. Theorem 2 stated in [4] and recalled below gives a characterization by considering the left-inverse dynamical matrices.

Theorem 2 ([4]): An output $y_{k}$ of the system (1) assumed to be square, with left inherent delay $r$, is a flat output if there exists a positive integer $K<\infty$ such that, for all sequences in $\mathcal{J}^{r+K}$, the following equality, involving the product of left-inverse dynamical matrices, applies for all $k \geq 0$ :

$P_{\sigma(k+K-1: k+K-1+r)} P_{\sigma(k+K-2: k+K-2+r)} \cdots P_{\sigma(k: k+r)}=\mathbf{0}$

The point is that the computational cost of the test (8) grows exponentially with respect to the number $K$ of matrices involved in (8). Besides, no upper bound for $K$ is given. The first objective of the paper is to propose an alternative condition with a reduction of the computational cost. As the condition only involves the left-inverse dynamical matrices of the left $r$-delay inverse system, we define below an auxiliary system and rewrite Theorem 2 .

\section{B. Auxiliary system}

Let us define the auxiliary system of (1) as the switched linear system given by

$$
q_{k+1}=Q_{\sigma^{\prime}(k)} q_{k}
$$

with $q_{k} \in \mathbb{R}^{n}$ and $\sigma^{\prime}$ a switching rule defined as follows. Consider the mapping $\phi: \mathcal{J}^{r+1} \rightarrow \mathcal{H}=\left\{1, \ldots, J^{r+1}\right\}$ that assigns to each possible sequence $\{\sigma(k), \ldots, \sigma(k+r)\}$ an integer $h$ from the set $\mathcal{H}$ which uniquely identifies the sequence. Then, the switching rule $\sigma^{\prime}$ is defined as the function from $\mathbb{N}$ to $\mathcal{H}$ which associates to each integer $k \in \mathbb{N}$ the quantity $\sigma^{\prime}(k)=\phi(\sigma(k), \ldots, \sigma(k+r)) \in \mathcal{H}$. The value $\sigma^{\prime}(k)$ is the mode of the auxiliary switched linear system (9) and $Q_{\sigma^{\prime}(k)}=P_{\sigma(k: k+r)}$. We denote by $\mathcal{Q}$ the set of all the matrices $Q_{h}(h \in \mathcal{H})$.

By considering the auxiliary system (9), we are now in position of reformulating Theorem 2 which turns into

Theorem 3: An output $y_{k}$ of the system (1) assumed to be square, with left inherent delay $r$, is a flat output if there exists a positive integer $K<\infty$ such that, for all admissible sequences $\left\{\sigma^{\prime}(k), \ldots, \sigma^{\prime}(k+K-1)\right\} \in \mathcal{H}^{K}$, the following equality, involving the product of the dynamical matrices of the auxiliary system (9), applies for all $k \geq 0$ :

$$
Q_{\sigma^{\prime}(k+K-1)} Q_{\sigma^{\prime}(k+K-2)} \cdots Q_{\sigma^{\prime}(k)}=\mathbf{0}
$$

Proof: The proof is a straightforward consequence of the definition of the auxiliary system.

We are now in position to give an alternative condition to (8). It is based on the consideration of (10) and the notion of nilpotent semigroups as presented in the next section. 


\section{FLAT OUTPUT CHARACTERIZATION AND NILPOTENT SEMIGROUPS}

\section{A. Main result}

Basics related to nilpotent semigroups are first recalled.

Definition 3 (Semigroup): A semigroup $\mathcal{S}$ is a set together with an associative internal law.

$\mathcal{S}$ is said to be finite if it has a finite number of elements. If $\mathcal{S}$ is a set of matrices, the associative internal law is the matrix multiplication. We denote by 0 the absorbing element of a semigroup when it exists.

Definition 4 (Nilpotent semigroup): A semigroup $\mathcal{S}$ with an absorbing element 0 is said to be nilpotent if there is an integer $t \in \mathbb{N}^{*}$ such that the internal law applied to any $t$ elements of $\mathcal{S}$ is always equal to 0 . The smallest integer $t$ is called the nilpotency class of $\mathcal{S}$.

If $\mathcal{S}$ is a set of matrices, applying the internal law to any $t$ elements of $\mathcal{S}$ amounts to performing the product of $t$ matrices of $\mathcal{S}$. The absorbing element is, in this case, the null matrix. Here, the elements of $\mathcal{S}$ are the matrices of $\mathcal{Q}$.

Theorem 4: If the matrices of $\mathcal{Q}$ of the auxiliary system (9) generate a nilpotent semigroup, then $y_{k}$ is a flat output.

Proof: If the matrices of $\mathcal{Q}$ of the auxiliary system (9) generate a nilpotent semigroup, by definition, for any $t$-uple $\left(h_{1}, \ldots, h_{t}\right) \in \mathcal{H}^{t}, t$ being the class of nilpotency of $\mathcal{Q}$, one has

$$
\prod_{i=1}^{t} Q_{h_{i}}=\mathbf{0}
$$

Hence, (10) is fulfilled with $K=t$. As a result, Theorem 3 holds and means that $y_{k}$ is a flat output.

Corollary 1: If the matrices of $\mathcal{Q}$ generate a nilpotent semigroup, the integer $K$ is finite and is upper bounded by the dimension $n$ of the system (1).

Proof: If the matrices of $\mathcal{Q}$ generate a nilpotent semigroup, the integer $K$ is equal to the class of nilpotency $t$ of the semigroup. The class of nilpotency being actually bounded by the dimension of the matrices involved in the semigroup, $K$ is bounded by the dimension of the matrices of $\mathcal{Q}$, that is precisely $n$, the dimension of the system (1).

Remark 2: A necessary condition for the matrices of $\mathcal{Q}$ to generate a nilpotent semigroup is that all the matrices of $\mathcal{Q}$ are nilpotent, that is all their eigenvalues are zero. Indeed, (11) must hold in particular for the $t$-uples $\left(h_{1}, \ldots, h_{t}\right)$ with $h_{i}=h_{l}$ for all $i, l \in\{1, \ldots, t\}$.

Remark 3: It is worth pointing out that different sequences $\{\sigma(k), \ldots, \sigma(k+r)\}$ of (1) and so different modes $\sigma^{\prime}(k)=\phi(\sigma(k), \ldots, \sigma(k+r))$ of (9) might lead to identical matrices $Q_{\sigma^{\prime}(k)}$. As a result, $\mathcal{Q}$ is a multiset ${ }^{1}$ and we should consider only distinct matrices of $\mathcal{Q}$ to reduce the computational cost. We denote by $\mathcal{Z}$ the set of distinct matrices of $\mathcal{Q}$ and by $L$ its cardinality. Its elements are denoted by $Z_{l}(l=1, \ldots, L)$. Clearly, $\mathcal{Z} \subseteq \mathcal{Q}, L \leq J^{r+1}$ and Theorem 4 still applies by considering $\mathcal{Z}$ instead of $\mathcal{Q}$.

\footnotetext{
${ }^{1}$ The notion of multiset is a generalization of the notion of set in which elements are allowed to appear more than once.
}

\section{B. Equivalence on conditions}

Let us first point out that the switching rule $\sigma^{\prime}$ of the auxiliary system (9) is constrained. Indeed, since $\sigma^{\prime}(k)=$ $\phi(\sigma(k), \ldots, \sigma(k+r))$ and $\sigma^{\prime}(k+1)=\phi(\sigma(k+1), \ldots, \sigma(k+$ $r+1)), \sigma^{\prime}(k)$ and $\sigma^{\prime}(k+1)$ depend on the common subsequence $\{\sigma(k+1), \ldots, \sigma(k+r)\}$ and thereby are related one another. Hence, even in the case when the switching rule $\sigma$ of (1) is arbitrary, given a matrix $Q_{\sigma^{\prime}(k)}=P_{\sigma(k: k+r)}$, the matrix $Q_{\sigma^{\prime}(k+1)}=P_{\sigma(k+1: k+1+r)}$ is constrained. To formalize this constraint, it is convenient to introduce a socalled set of feasible transitions.

Definition 5: The set $\Gamma\left(\sigma^{\prime}(k)\right)$ of feasible transitions from mode $\sigma^{\prime}(k)$ is the set defined by

$$
\begin{aligned}
\Gamma\left(\sigma^{\prime}(k)\right)= & \{h \in \mathcal{H}: h=\phi(\sigma(k+1), \ldots, \\
& \sigma(k+r+1)), \forall \sigma(k+r+1) \in \mathcal{J}\}
\end{aligned}
$$

In other words, $\Gamma\left(\sigma^{\prime}(k)\right)$ is the set $h \in \mathcal{H}$ which can be reached when $\sigma(k+r+1)$ varies over the whole range $\mathcal{J}$, $\sigma^{\prime}(k)$ and so the sequence $\{\sigma(k+1), \ldots, \sigma(k+r)\}$ being imposed. One has $\Gamma\left(\sigma^{\prime}(k)\right) \subseteq \mathcal{H}$ which clearly formalizes that $\sigma^{\prime}$ is constrained. It is clear that $\Gamma\left(\sigma^{\prime}(k)\right)$ can never be the empty set.

Definition 6: A sequence $\left\{h_{1}, h_{2}, \ldots\right\}$ is said admissible if for any $i \geq 0$

$$
h_{i+1} \in \Gamma\left(h_{i}\right)
$$

Let us introduce the map $\mu: \mathcal{H} \rightarrow \mathcal{Q}$ which assigns to each integer $h \in \mathcal{H}$ the matrix $Q_{h} \in \mathcal{Q}$. The restriction of $\mu$ to a particular subset $\Gamma(h)$ of $\mathcal{H}$ is denoted by $\mu_{\Gamma(h)}$.

Definition 7: A sequence of matrices $\left\{Q_{h_{1}}, Q_{h_{2}}, \ldots\right\}$ $\left(h_{i} \in \mathcal{H}\right)$ is said admissible if for any $h_{i} \in \mathcal{H}$

$$
Q_{h_{i+1}} \in \mathcal{R}\left(\mu_{\Gamma\left(h_{i}\right)}\right)
$$

where the notation $\mathcal{R}$ denotes the range of the function. The following proposition applies:

Proposition 1: The conditions (10) and (11) are equivalent if and only if

$$
\forall h_{i} \in \mathcal{H}, \mathcal{R}\left(\mu_{\Gamma\left(h_{i}\right)}\right)=\mathcal{Q}
$$

Proof: The statement (11) $\Rightarrow$ (10) is always true regardless of the condition (15). Still, it must be shown that (10) implies (11) provided that (15) is fulfilled. The condition $\forall h_{i}, \mathcal{R}\left(\mu_{\Gamma\left(h_{i}\right)}\right)=\mathcal{Q}$ means that, for any arbitrary mode $h_{i} \in \mathcal{H}, Q_{h_{i+1}}$ can be any matrix in $\mathcal{Q}$. Hence, for any $t$-uple $\left(h_{1}, \ldots, h_{t}\right)$, the sequence $\left\{Q_{h_{1}}, \ldots, Q_{h_{t}}\right\}$ is an admissible sequence for (9). Finally, the set of products $Q_{h_{1}} \cdots Q_{h_{t}}$ for all $t$-uples $\left(h_{1}, \ldots, h_{t}\right)$ coincides with the set of products (10) for all $k \geq 0$. That completes the proof.

Similarly to Remark 3, if different sequences $\{\sigma(k), \ldots, \sigma(k+r)\}$ and so different $\sigma^{\prime}(k)=$ $\phi(\sigma(k), \ldots, \sigma(k+r))$ lead to identical matrices $Q_{\sigma^{\prime}(k)}$, Proposition 1 still can be applied with a lower computational cost if the multiset $\mathcal{Q}$ is replaced by the set $\mathcal{Z}$ of distinct elements of $\mathcal{Q}$. Hence, we will consider hereafter the set $\mathcal{Z}$.

Remark 4: It can be easily seen that (15) is always satisfied for at least two particular cases: when the inherent delay 
$r$ is equal to zero or if it is equal to one and that $C$ does not depend on $\sigma$. These two cases encompass a large class of systems and we will illustrate in Section V that (15) also applies in other cases.

\section{Computational issues}

In this section, we propose an algorithm that allows to check whether or not a set of matrices generates a nilpotent semigroup that is, if Theorem 4 is fulfilled. It is shown that it has a polynomial complexity and is theoretically motivated by Levitsky's theorem (Theorem 2.1.7 stated in [7]).

Theorem 5 (Levitsky's theorem): Any semigroup of nilpotent matrices can be triangularized.

In other words, all the matrices of a same nilpotent semigroup can be rewritten as upper triangular matrices with zeros on the diagonal up to a common change of basis. The consequence of this theorem is central for our purpose. Indeed, determining whether or not the matrices of $\mathcal{Z}$ generate a nilpotent semigroup amounts to checking whether or not $\mathcal{Z}$ can be simultaneously triangularized. It is a necessary and sufficient condition. The approach we propose to check Theorem 5 is inspired from the general triangularization method provided in [8] and corresponds to Algorithm 1. Next, some peculiarities that apply to our special case will be addressed to provide a fully-specified algorithm for flat output characterization.

The algorithm is expected to return a change of basis $S$ that simultaneously triangularizes the set $\mathcal{Z}$ composed of the $L$ matrices $Z_{l}, l \in\{1, \ldots, L\}$ of dimension $n$. According to Remark $2, Z_{l}, l \in\{1, \ldots, L\}$ are assumed to be nilpotent.

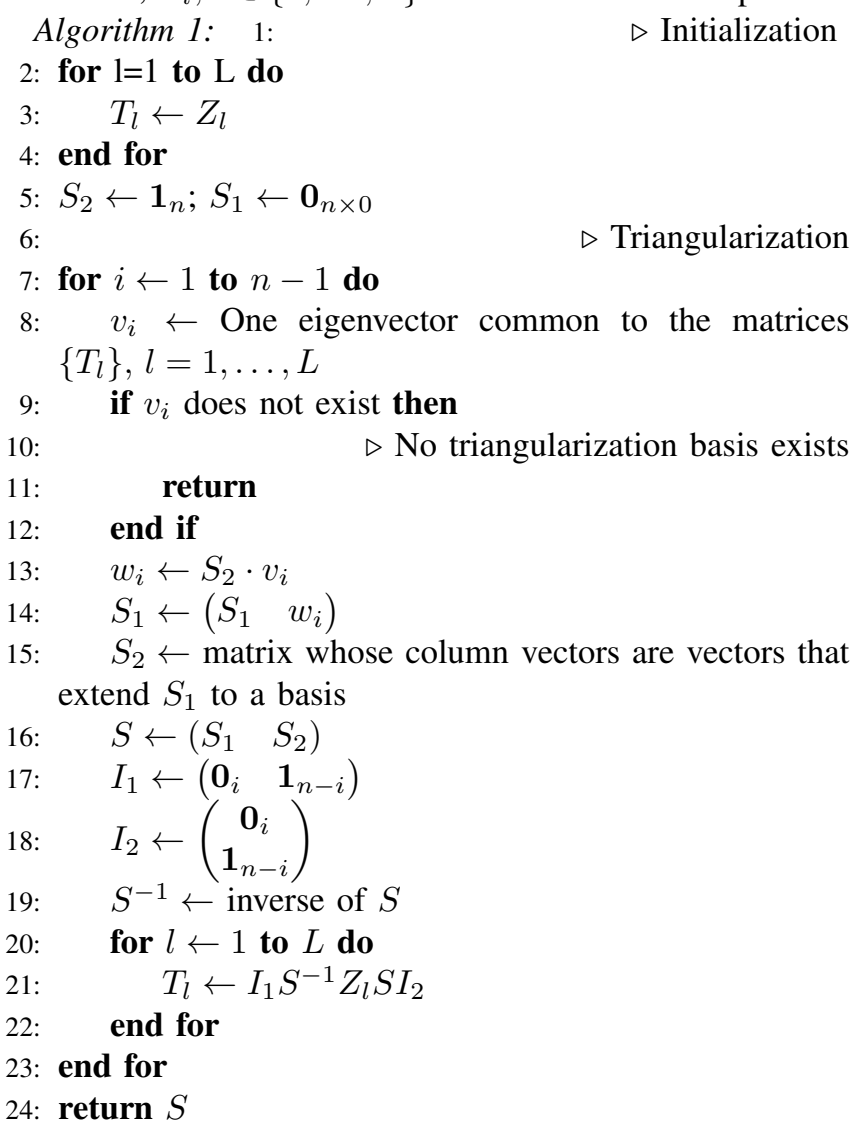

The following comments are in order:

- Line 1 to Line 5 corresponds to the initialization. Matrices $T_{l}(l=1, \ldots, L)$ play the role of the auxiliary variables and are initialized at the beginning with the matrices $Z_{l}$ of the set $\mathcal{Z}$.

- At most $n-1$ successive loops from Line 7 to Line 23 are performed as stressed by Corollary 1 .

- Line 8 corresponds to the first step of a given loop $i$. It consists in finding out a eigenvector $v_{i}$ which is common to the matrices $T_{l}(l=1, \ldots, L)$. Consequently, in the first loop, $v_{1}$ is a common eigenvector of the matrices $Z_{l}$ of $\mathcal{Z}$. It is worth pointing out that if this step fails in the loop $i$, it means that the matrices $T_{l}$ $(l=1, \ldots, L)$ do not have any common eigenvector $v_{i}$ and the algorithm stops. Levitsky's theorem stating a necessary and sufficient condition, it can be concluded that $\mathcal{Z}$ does not generate a nilpotent semigroup and Theorem 4 is not fulfilled.

- Line 14 describes the fact that the final change of basis $S$ is built column after column. Each new vector $v_{i}$ carried out in the loop $v_{i}$ is added (actually after a change of basis notified at Line 13) resulting in a matrix $S_{1}=\left(v_{1} \cdots v_{i}\right)$. When $i=n$ then $S=\left(S_{1} S_{2}\right), S_{1}$ and $S_{2}$ resulting from the loop $n-1$.

- At Line 15, $S_{1}$ must be extended to a basis. By extension, it is meant a set of $n-i$ vectors $w_{j}$ so that $S=\left(S_{1} w_{1} \cdots w_{n-i}\right)$ is full rank. The matrix $S_{2}$ is precisely $S_{2}=\left(w_{1} \cdots w_{n-i}\right)$.

- Line 21 performs the current change of basis $S$ to the matrices $Z_{l}$. The multiplications by $I_{1}$ and $I_{2}$ merely corresponds to the extraction of a square matrix of dimension $n-i$ from the matrix $S^{-1} Z_{l} S$, that the first $i$ rows and columns of $S^{-1} Z_{l} S$ are removed. A new set of matrices $T_{l}$ is thereby obtained. A new loop can restart from Line 7.

Such an algorithm is quite general. However, the determination of a common eigenvector at Line 8 and the extension to a basis at Line 15 can be particularized to our special context.

1) Determination of a common eigenvector: According to Remark 2, a necessary condition for the set $\mathcal{Z}$ to generate a nilpotent semigroup is that all the matrices $Z_{l}$ of $\mathcal{Z}$ are nilpotent, that is all their eigenvalues are zero. The set $\mathcal{T}=\left\{T_{l}\right\}, l=1, \ldots, L$ corresponds exactly to $\mathcal{Z}$ at the initialization and it is updated at each loop $i$ through a linear change of basis $S$ at Line 21 which preserves the eigenvalues. Hence, the eigenvalues of the $T_{l}$ 's are all zero whatever the loop $i \geq 0$ is. Consequently, for any $v_{i}(i=1, \ldots, n)$ and $\forall T_{l} \in \overline{\mathcal{T}}, T_{l} v_{i}=0$ holds. Hence, $v_{i}$ is a non zero solution of

$$
R v_{i}=\mathbf{0} \text { with } R=\left(\begin{array}{c}
T_{1} \\
\vdots \\
T_{L}
\end{array}\right)
$$

As a result, $v_{i}$ is a non zero vector of the null space of $R$ denoted $\operatorname{ker}(R)$. 
2) Extension to a basis: At Line 15, $S_{1}$ must be extended to a basis. We must thereby find out a set of $n-i$ vectors $w_{j}$ so that $S=\left(\begin{array}{llll}S_{1} & w_{1} & \cdots & w_{n-i}\end{array}\right)$ is full rank. It can be obtained by determining a basis of the kernel of the transpose of $S_{1}$. In other words, $S_{2}=\left(w_{1} \cdots w_{n-i}\right)$ can be any basis of $\operatorname{ker}\left(S_{1}^{\prime}\right)$ where the symbol ' stands for transposition.

\section{Complexity}

All the operations can easily be performed by software involving usual built-in functions. The complexity of the condition (8) is $O\left(J^{r+K} K n^{3}\right)$. The point is that the complexity is exponential with respect to the number of matrices $K$ involved in the product (8), which can be large. On the other hand, let us assess the computational cost of the flat output characterization approach based on nilpotent semigroups by examining Algorithm 1. Considering a given loop, the most complex operations are performed at Lines 8, 15, 19 and 21. Lines 8 and 15 consist in determining the kernel of a matrix. It is usually based on singular value decomposition of a $\mathbb{R}^{a \times b}$ matrix for which known algorithms with complexity $O\left(4 a b^{2}+8 b^{3}\right)$ exist. Line 19 has complexity $O\left(n^{3}\right)$ when considering usual inversion algorithms. Line 21 is a change of basis. The multiplications by $I_{1}$ and $I_{2}$ can be avoided since its purpose is merely to extract a square matrix of dimension $n-i$ from the matrix $S^{-1} Z_{l} S$. Therefore, the two operations to be considered are the two matrix multiplications of $Z_{l}$ by $S$ and $S^{-1}$. Matrix multiplications have complexity at most $O\left(n^{3}\right)$. Therefore, the complexity of Lines 20 to 22 is $O\left(L n^{3}\right)$ or $O\left(J^{r+1} n^{3}\right)$ due to the inequality $L \leq J^{r+1}$ (recall Remark 3). This part of the code is the one with the largest complexity. The operations are repeated over at most $n$ loops. Therefore, the global complexity of Algorithm 1 is $O\left(J^{r+1} n^{4}\right)$. There is no more exponential complexity with respect to the number of matrices involved in the product (8).

It is an improvement insofar as the complexity is no longer exponential with respect to the parameter $K$.

\section{EXTENSION TO LPV SYSTEMS}

The results stated in the previous sections can be interestingly extended in a rather straightforward way to LPV systems. Indeed, the system (1) can be viewed as an LPV system as soon as we consider that the switching rule $\sigma$ is replaced by a function which takes values in a continuum. If so, the sets $\mathcal{J}, \mathcal{H}, \Gamma\left(\sigma^{\prime}(k)\right), \mathcal{Q}$ and $\mathcal{Z}$ must be considered as uncountable sets. The $r$-delay inverse system (5), the auxiliary system (9) together with the mapping $\phi$ still make sense as soon as $\sigma^{\prime}$ is considered as a function, similarly to $\sigma$, taking values in a continuum. Besides and most importantly, it turns out that both semigroups (Definition 3) and the nilpotent semigroups (Definition 4) are still well defined for an uncountable set $\mathcal{S}$. As a result, Theorem 4 still applies.

On the other hand, Levitsky's theorem, which allows for checking whether Theorem 4 is fulfilled, applies for any semigroup, including semigroups with infinite cardinality, which is precisely the case here. It is recalled that Levitsky's theorem asserts that " $\mathcal{Z}$ generates a nilpotent semigroup if the matrices of $\mathcal{Z}$ can be simultaneously triangularized". The key point is that, considering $\mathcal{Z}$ as an uncoutable set of matrices, it can be reformulated in a strictly equivalent way stating that "the matrices $Z_{\sigma^{\prime}(k)}$ must be simultaneously triangularized with a change of basis that does not depend on $\sigma^{\prime}(k)$ ". Taking into acount the aforementionned considerations and combining Theorem 4 and Theorem 5, the following theorem holds for characterizing flat outputs of LPV systems

Theorem 6: If the matrices of $\mathcal{Z}$ of the auxiliary system (9) can be simultaneously triangularized independently of $\sigma^{\prime}(k)$, then $y_{k}$ is a flat output.

Algorithm 1 still applies up to some minor modifications. The loops at Line 2 and Line 20 can be removed or equivalently, $L$ can be set to $L=1$. Besides, the determination of a common eigenvector at Line 8 , that is the search for a non zero vector $v_{i}$ of $\operatorname{ker}(R)$ as explained in Subsection IIIC.1 turns into the search for a non zero vector $v_{i}$ of $\operatorname{ker}\left(T_{1}\right)$ (since $L=1$ and so $R=T_{1}$ ) independent of $\sigma^{\prime}(k)$. Line 8 has to be replaced by

8: $v_{i} \leftarrow$ One eigenvector of $T_{1}$ independent of $\sigma^{\prime}(k)$

The flat output characterization based on nilpotent semigroups for LPV systems is valuable for two major reasons. First, flat outputs characterization of LPV systems has never been addressed so far in the literature. Secondly, the characterization through (8) or equivalently (3) cannot be done for LPV systems since that requires to check an infinite number of possible products. Indeed, $\sigma$ taking values in a continuum, the number of sequences $\{\sigma\}$ in Theorem 2 or sequences $\left\{\sigma^{\prime}\right\}$ in Theorem 3 would be infinite.

\section{ILLUSTRATIVE EXAMPLES}

\section{A. Example 1: switching systems}

Consider the SISO switched linear system of the form (1). The dimension is $n=4$, the switching rule $\sigma$, not detailed here, is assumed to deliver arbitrary sequences and the number of modes is $J=3$. According to the mode, the state space matrices numerically read

$$
\begin{gathered}
A_{1}=\left(\begin{array}{cccc}
-1 & -0.5 & -0.5 & 0 \\
1 & 1.5 & 1.5 & 0 \\
1 & 0.5 & 0.5 & 1 \\
1 & 0 & 1 & 0
\end{array}\right), A_{2}=\left(\begin{array}{cccc}
-1 & -1 & -1 & -0.5 \\
1 & 2 & 2 & 0.5 \\
1 & 1 & 1 & 1.5 \\
1 & 0 & 1 & 0
\end{array}\right), \\
A_{3}=\left(\begin{array}{cccc}
-1 & -2.5 & -2.5 & -2 \\
1 & 3.5 & 3.5 & 2 \\
1 & 2.5 & 2.5 & 3 \\
1 & 0 & 1 & 0
\end{array}\right) \\
B_{1}=B_{2}=B_{3}=\left(\begin{array}{llll}
0 & 0 & 0 & 1
\end{array}\right)^{\prime} \\
C_{1}=C_{2}=C_{3}=\left(\begin{array}{llll}
2 & 1 & 1 & 0
\end{array}\right) \\
D_{1}=D_{2}=D_{3}=0
\end{gathered}
$$

Since it is a SISO system, that for all $i \in \mathcal{J}, D_{i} \neq 0$, for all $(i, j) \in \mathcal{J}^{2}, C_{i} B_{j}=0$ and that for all $(i, j, l) \in$ $\mathcal{J}^{3}, C_{i} A_{j} B_{l} \neq 0$, the inherent delay is $r=2$. Let us derive 
the corresponding auxiliary system as defined in Section IIB. To this end, we must define the mapping $\phi$. The number of possible sequences over any interval of time $[k: k+r]$ is $J^{r+1}=3^{2+1}=27$ and

$$
\begin{array}{cc}
\phi(\{1,1,1\})=1 & \cdots \\
\phi(\{1,1,2\})=2 & \phi(\{3,3,1\})=25 \\
\phi(\{1,1,3\})=3 & \phi(\{3,3,2\})=26 \\
\cdots & \phi(\{3,3,3\})=27
\end{array}
$$

The set $\Gamma$ of feasible transitions obeys

$$
\begin{array}{ll}
\Gamma(1)=\{1,2,3\} & \cdots \\
\Gamma(2)=\{4,5,6\} & \Gamma(26)=\{23,24,25\} \\
\ldots & \Gamma(27)=\{25,26,27\}
\end{array}
$$

It turns out that the multiset $\mathcal{Q}$ has $L=3$ distinct matrices and the matrices $Z_{l}$ of the corresponding set $\mathcal{Z}$ numerically read

$$
\begin{gathered}
Z_{1}=\left(\begin{array}{rrrr}
-1 & -0.5 & -0.5 & 0 \\
1 & 1.5 & 1.5 & 0 \\
1 & 0.5 & 0.5 & 1 \\
-2 & -2 & -2 & -1
\end{array}\right), Z_{2}=\left(\begin{array}{rrrr}
-1 & -1 & -1 & -0.5 \\
1 & 2 & 2 & 0.5 \\
1 & 1 & 1 & 1.5 \\
-2 & -3 & -3 & -2
\end{array}\right) \\
Z_{3}=\left(\begin{array}{rrrr}
-1 & -2.5 & -2.5 & -2 \\
1 & 3.5 & 3.5 & 2 \\
1 & 2.5 & 2.5 & 3 \\
-2 & -6 & -6 & -5
\end{array}\right)
\end{gathered}
$$

One has

$$
\mu(\Gamma(1))=\cdots=\mu(\Gamma(27))=\left\{Z_{1}, Z_{2}, Z_{3}\right\}
$$

Hence, condition (15) of Proposition 1 is fulfilled and so applies beyond the particular cases mentioned in Remark 4. Finally, it turns out that Algorithm 1 succeeds and returns the following change of basis $S$

$$
S=\left(\begin{array}{crrc}
0 & 0.3780 & -0.9258 & 0 \\
0.7071 & -0.3780 & -0.1543 & 0.5774 \\
-0.7071 & -0.3780 & -0.1543 & 0.5774 \\
0 & 0.7559 & 0.3086 & 0.5774
\end{array}\right)
$$

As a consequence, based on Theorem 4 and Theorem 5, we conclude that $y_{k}$ is a flat output.

\section{B. Example 2: LPV systems}

We investigate an LPV system given by the following form $^{2}$

$$
\left\{\begin{array}{l}
x_{k+1}=A_{\sigma(k)} x_{k}+B_{\sigma(k)} u_{k} \\
y_{k}=C_{\sigma(k)} x_{k}+D_{\sigma(k)} u_{k}
\end{array}\right.
$$

where $x_{k} \in \mathbb{R}^{4}, u_{k} \in \mathbb{R}, y_{k} \in \mathbb{R}$ and the state space matrices numerically read

$$
A_{\sigma(k)}=\left(\begin{array}{cccc}
0 & 1 & 0 & 0 \\
1 & 0 & 1 & 0 \\
0 & 0 & 0 & 1 \\
\sigma_{k}^{(1)} & \sigma_{k}^{(2)} & 1 & 0
\end{array}\right), B_{\sigma(k)}=\left(\begin{array}{l}
0 \\
0 \\
1 \\
0
\end{array}\right),
$$

\footnotetext{
${ }^{2}$ In the framework of LPV systems, the notation $X\left(\rho_{k}\right)$ is often used and refers to matrices which depend on a time-varying parameter $\rho_{k}$. Hence here, by $X_{\sigma(k)}$, it must be understood a matrix $X$ which depends on a time-varying parameter $\sigma(k)$ with $\sigma(k)$ taking values in a continuum. $\sigma_{k}^{(i)}$ will denote the $i$-th component of $\sigma(k)$
}

$$
C_{\sigma(k)}=\left(\begin{array}{llll}
1 & 0 & 1 & 0
\end{array}\right), D_{\sigma(k)}=0
$$

As stressed in Section IV, $\sigma$ must be considered here as a function which takes values in a continuum. Since it is a SISO system, that $D_{\sigma(k)}=0$ for all $k$ and that $C_{\sigma(k+1)} B_{\sigma(k)}=1$ independently of $\sigma$, the inherent delay is 1 . The set $\mathcal{Q}$ of the dynamical matrices of the left-inverse system (5) are the matrices $Q_{\sigma^{\prime}(k)}=P_{\sigma(k: k+1)}$ which read for all $\sigma^{\prime}(k)=\{\sigma(k), \sigma(k+1)\}$

$$
Q_{\sigma^{\prime}(k)}=\left(\begin{array}{cccc}
0 & 1 & 0 & 0 \\
1 & 0 & 1 & 0 \\
0 & -1 & 0 & 0 \\
\sigma_{k}^{(1)} & \sigma_{k}^{(2)} & 1 & 0
\end{array}\right)
$$

It turns out that Theorem 6 is fulfilled with a triangularization basis $S$ which numerically reads

$$
S=\left(\begin{array}{cccc}
0 & 1 & 0 & 1 \\
0 & 0 & 1 & 0 \\
0 & -1 & 0 & 0 \\
1 & 0 & 0 & 0
\end{array}\right)
$$

Finally, since $Q_{\sigma^{\prime}(k)}$ only depends on $\sigma^{\prime}$ at time $k$, it is clear that condition (15) of Proposition 1 is fulfilled, guaranteeing that conditions (10) and (11) are equivalent. The interest of the result stated in this paper is that (11) provides an alternative to (10), hence solving the intractability of (10) for LPV systems.

\section{CONCLUSION}

We have provided a condition to characterize flat outputs of switched linear discrete-time systems. Then, the condition is extended to LPV discrete-time systems. The condition is based on the notion of nilpotent semigroups. A tractable fully-specified algorithm, with polynomial complexity with respect to the dimension of the system, has been provided to check the condition.

\section{REFERENCES}

[1] M. Fliess, J. Levine, P. Martin, and P. Rouchon. Flatness and defect of non-linear systems: introductory theory and examples. Int. Jour. of Control, 61(6):1327-1361, 1995.

[2] H. Sira-Ramirez and S. K. Agrawal. Differentially Flat Systems. Marcel Dekker, New York, 2004.

[3] J. Levine and D. V. Nguyen. Flat output characterization for linear systems using polynomial matrices. Systems and control Letters, 48:69$75,2003$.

[4] G. Millérioux and J. Daafouz. Flatness of switched linear discrete-time systems. IEEE Trans. on Automatic Control, 54(3):615-619, March 2009.

[5] L. Vu and D. Liberzon. Invertibility of switched linear systems. Automatica, 44(4):949-958, 2008.

[6] S. Sundaram and C. Hadjicostis. Designing stable inverters and state observers for switched linear systems with unknown inputs. In Proc. of the 45th IEEE Conference on Decision and Control, San Diego, CA, USA, December 2006.

[7] Heydar Radjavi and Peter Rosenthal. Simultaneous Triangularization. Springer, 2000.

[8] C. Dubi. An algorithmic approach to simultaneous triangularization. Linear Algebra and its Applications, 430(11-12):2975 - 2981, 2009. 